\title{
Lymph node metastases in thymic tumors: The more we know, the less we know
}

\author{
Usman Ahmad, MD, and Siva Raja, MD, PhD
}

\footnotetext{
From the Department of Cardiothoracic Surgery, Section of Thoracic Surgery, Heart and Vascular Institute, Cleveland Clinic, Cleveland, Ohio.

Disclosures: Authors have nothing to disclose with regard to commercial support.

Received for publication March 20, 2017; accepted for publication March 21, 2017; available ahead of print April $27,2017$.

Address for reprints: Usman Ahmad, MD, Department of Cardiothoracic Surgery, Heart and Vascular Institute, Cleveland Clinic, Cleveland, OH 44195 (E-mail: ahmadu@ccf.org).

J Thorac Cardiovasc Surg 2017;154:e15-6

$0022-5223 / \$ 36.00$

Copyright (C) 2017 Published by Elsevier Inc. on behalf of The American Association for Thoracic Surgery http://dx.doi.org/10.1016/j.jtcvs.2017.03.108
}

The field of thymic tumors suffers from profound lack of prospective studies. The few available clinical trials accrued limited numbers of patients despite long study periods. ${ }^{1,2}$ Questions regarding extent of resection, use of neoadjuvant and adjuvant treatment, radiographic surveillance for recurrence, and certainly management of recurrences are addressed largely by retrospective data and expert consensus. Relatively long recurrence freeintervals also make it difficult to study primary and recurrent thymomas prospectively in the same series.

One of biggest challenges, however, is the heterogeneity in practice among various institutions. Differences in treatment and surveillance paradigms make it difficult to compare outcomes. Similarly, adoption of new recommendations is much slower than with other malignancies. A case in point is the issue of lymph node assessment in the treatment of thymic tumors. In this issue of the Journal, Bertoglio and colleagues ${ }^{3}$ have described the case of a 57-year-old woman who underwent resection of a $9-\mathrm{cm}$ mediastinal mass without preoperative tissue diagnosis or positron emission tomography. Interestingly, paratracheal lymph node sampling was performed. The pathologic evaluation showed a Masaoka stage III, World Health Organization type B1 thymoma. The patient underwent computed tomographic surveillance until 10 years later, when she was noted to have fludeoxyglucose F 18 (INN fludeoxyglucose [18F])-avid right paratracheal lymphadenopathy. A biopsy sample was obtained through mediastinoscopy and was consistent with recurrent thymoma. Further 27 Gy of radiation treatment was delivered to the site of recurrence. Bertoglio and colleagues ${ }^{3}$ are to be lauded for successfully conducting long-term follow-up and identifying the recurrence.

Systematic lymph node sampling during thymic tumor resection has not been standard practice, especially outside Japan. This is likely because of the low anecdotally reported rate of lymph node metastasis and recurrences. In this case, it is unclear whether the paratracheal lymph node was enlarged on the preoperative computed tomographic scan;

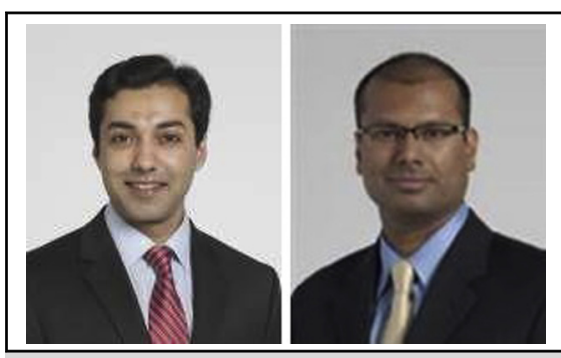

Usman Ahmad, MD (left), and Siva Raja, MD, PhD (right)

Central Message

Lymph node metastasis and recurrence are uncommon in thymomas. Systematic nodal sampling is recommended for locally advanced tumors. Prospective data are needed to study these tumors more effectively.

See Article page e11.

however, Bertoglio and colleagues ${ }^{3}$ chose to perform sampling at the time of resection. If this was an effort at nodal sampling, the reasoning behind the choice of sampled stations is unclear.

Systematic lymph node sampling is now encouraged, and certainly the newly proposed TNM staging system relies on accurate nodal staging. In the review of international data, most institutions did not routinely assess lymph nodes at resection. ${ }^{4}$ The most complete data on nodal involvement come from retrospective review of the Japanese Association for Research in the Thymus (JART) registry. In a series of 1064 thymomas, lymph node metastases were noted in only $2 \%$ of patients; however, $6 \%$ of patients with stage III thymoma had lymph node involvement. Among nodepositive patients, $89 \%$ had anterior mediastinal perithymic nodal involvement, whereas $26 \%$ had deep intrathoracic nodal metastases. The rate of lymph node metastasis was higher in thymic carcinoma $(27 \%)$ and neuroendocrine tumors $(28 \%))^{5}$ Patients with nodal involvement had significantly worse overall survival.

On the basis of pooled analysis of international data, a recommendation has been made for complete resection of perithymic tissue to include all nodal tissue and for systematic sampling of intrathoracic nodes in locally advanced thymic tumors. It is unclear, however, how best to stage the intrathoracic nodes in this high-risk population. These retrospective analyses are certainly a great stride forward 
and have increased our knowledge, but they have also led to more questions. Thymomas are anterior mediastinal tumors, and in theory they should have equal predilection to metastasize to right- and left-sided intrathoracic and paratracheal lymph nodes. Does that mean we should consider submitting high risk patients to transbronchial biopsy or mediastinoscopy to assess the mediastinum appropriately? Which nodal stations should be sampled? Similarly, what is the best surgical approach that would allow for systematic nodal sampling during resection with curative intent?

Although each of these questions has been addressed with a clinical trial in the lung cancer field, this is unlikely to ever be the case for thymic tumors. The strategy most likely to help address these issues is the prospective collection of uniform, high-quality data that may allow meaningful comparative analysis. The International Thymic Malignancy Interest Group has established an online prospective database for thymic tumors (www.ccehub.org/ portal/itmig). All institutions are encouraged to participate and populate the database, so that we may learn from our collective experience and make progress in the field of thymic tumors.

\section{References}

1. Kim ES, Putnam JB, Komaki R, Walsh GL, Ro JY, Shin HJ, et al. Phase II study of a multidisciplinary approach with induction chemotherapy, followed by surgical resection, radiation therapy, and consolidation chemotherapy for unresectable malignant thymomas: final report. Lung Cancer. 2004;44:369-79.

2. Korst RJ, Bezjak A, Blackmon S, Choi N, Fidias P, Liu G, et al. Neoadjuvant chemoradiotherapy for locally advanced thymic tumors: a phase II, multi-institutional clinical trial. J Thorac Cardiovasc Surg. 2014;147:36-44. 46.e1.

3. Bertoglio P, Lucchi M, Aprile V, Alì G, Mussi A. Single lymph-node metastasis 10 years after radical resection of a thymoma. J Thorac Cardiovasc Surg. 2017;154: e11-3.

4. Kondo K, Van Schil P, Detterbeck FC, Okumura M, Stratton K, Giroux D, et al; Staging and Prognostic Factors Committee; Members of the Advisory Boards; Participating Institutions of the Thymic Domain. The IASLC/ITMIG Thymic Epithelial Tumors Staging Project: proposals for the N and M components for the forthcoming (8th) edition of the TNM classification of malignant tumors. $J$ Thorac Oncol. 2014;9(9 Suppl 2):S81-7.

5. Kondo K, Monden Y. Lymphogenous and hematogenous metastasis of thymic epithelial tumors. Ann Thorac Surg. 2003;76:1859-64; discussion 1864-5. 